\title{
Stability Analysis Through the Direct Method of Lyapunov in the Oscillation of a Synchronous Machine
}

\author{
Carlos Alberto Abello Muñoz ${ }^{1}$, Pedro Pablo Cárdenas Alzate² \& Fernando Mesa ${ }^{2}$ \\ ${ }^{1}$ Universidad del Quindío, Facultad de Educación and GMME Research Group, Armenia, Colombia \\ ${ }^{2}$ Universidad Tecnológica de Pereira, Department of Mathematics and GEDNOL Research Group, Pereira, \\ Colombia \\ Correspondence: Pedro Pablo Cárdenas Alzate, Department of Mathematics, Universidad Tecnológica de Pereira, \\ Vereda la Julita. AA:97 - Código Postal: 660003, Pereira, Colombia. E-mail: ppablo@utp.edu.co
}

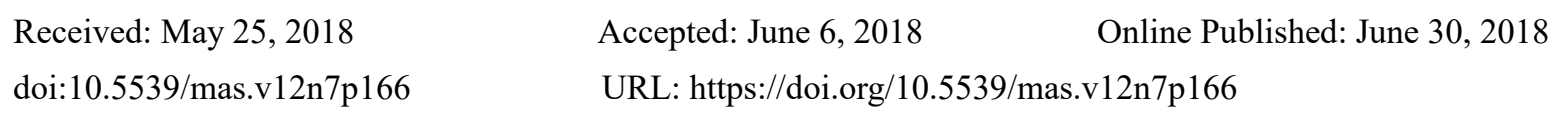

The research is financed by GEDNOL Research Group and GMME Research Group.

\begin{abstract}
This paper deals with the determination of the stability of a synchronous generator connected to an infinite bus when one of its parameters is varied. The direct method of Lyapunov is proposed for the construction of a scalar function that allows to characterize the stability of said system, this method is chosen since it has several practical advantages.
\end{abstract}

Keywords: synchronous generator, method of lyapunov, stability analysis

\section{Introduction}

The electrical power system can approach its stability limits due to the increase and variation of the energy demand, the generation capacity, the bad operation of automatic voltage regulators, the disconnection of some line or a generator, and different factors such as the quality regulations and environmental restrictions. Said stability refers to the ability of the power system to maintain the operating point on all buses in the system under normal conditions of operation.

There are several actions to try to improve the robustness of the system, one of them is the reinforcement of transmission lines between the generation and the load centers, the improvement in the control devices and the real-time communication (Khalil,1996). The main criterion of stability for synchronous machines is to maintain the speed of synchronization once the point of operation of the power system to which it is connected is altered, the time it takes to reach the new point of operation is known as transient, which is a key concept to define stability, since, if said transient is damped, then the system will be asymptotically stable in a finite period. This characteristic is desirable in all physical systems and is considered necessary for electrical power systems. (López, Sánchez, \& Moreno, 2014).

Among the different methods to analyze the stability of nonlinear dynamic systems, the most used is the direct method of Lyapunov, which not only allows the characterization of the point of equilibrium points, but also, through the functions of Lyapunov it is possible to analyze the regions of attraction of the system, transient states, estimation of the time of convergence and stability in finite time. The method is based on the existence of a function of the positive defined scalar type $\mathrm{V}$, such that its derivative along the trajectories is negative. The method establishes the conditions of existence of function $\mathrm{V}$ but does not address the procedure to determine this function. (Malisoff, \& Mazenc, 2009).

\section{Methodology}

We will try to find a Lyapunov function for the stability analysis of the dynamics of a synchronous generator connected to an infinite bus, for this we use the theory established by Lyapunov for the necessary conditions that the function that we want to find must fulfill. Then, we analyze the dynamics of the synchronous generator, its linearization around the point of operation and finally how a Lyapunov function can be determined from the linearized model. 


\subsection{Lyapunov Stability}

The theory of stability is of great importance in the analysis of dynamic engineering systems, where different problems such as stability at points of equilibrium (fixed points) or stability of input-output are analyzed. The characterization of stability at equilibrium points of the dynamic system is usually done using the stability theory of Lyapunov.

A point of equilibrium is said to be stable if system solutions that start close to that point remain close to it and are said to be asymptotically stable if, in addition, all solutions converge at that equilibrium point. Otherwise the point will be unstable. Mathematically, it is presented as follows:

Let the dynamic system be described by its state equations $\dot{x}=f(x)$ where $f: D \rightarrow \mathbb{R}^{n}$ is a map locally Lipschitz from a domain $D \subset \mathbb{R}^{n}$ in $\mathbb{R}^{n}$. Now, assuming $x^{*} \in D$ is a point of equilibrium, i.e., $f\left(x^{*}\right)=0$, then its classification is given by: (Seron, 2000)

- Stable if exists $\delta$ for all values of $\epsilon>0$ such that $\left\|x^{*}\right\|<\delta \Rightarrow\|x(t)\|<\epsilon$ for all $t \geq 0$.

- Unstable if it is not stable.

- Asymptotically stable if it is stable and $\delta$ can be chosen, such that $\left\|x^{*}\right\|<\delta \Rightarrow \lim _{t \rightarrow \infty} x(t)=0$

The stability of the equilibrium point can be determined by Lyapunov's theorem, which states that $V: D \rightarrow \mathbb{R}$ is a continuously differentiable function in the domain $D \subset \mathbb{R}^{n}$ that contains the origin, the derivative of $V$ along the the trajectories are given by:

$$
\dot{V}(\boldsymbol{x})=\sum_{i=1}^{n} \frac{\partial V}{\partial x_{i}} \dot{x}_{i}=\sum_{i=1}^{n} \frac{\partial V}{\partial x_{i}} f_{i}(x)
$$

Theorem: Let $x^{*}$ be a point of equilibrium in the domain $D \subset \mathbb{R}^{n}$. Let $V: D \rightarrow \mathbb{R}$ be completely differentiable such that: $V\left(\boldsymbol{x}^{*}\right)=0$ and $V(\boldsymbol{x})>0$ in $D-\{0\}$ and $\dot{V}(\boldsymbol{x}) \leq 0$ en $D$, then $x^{*}$ is a stable point. Also, if $\dot{V}(\boldsymbol{x}) \leq 0$ in $D-\{0\}$, then it will be asymptotically stable. (Strogatz, \& Fox, 1995)

Now, to determine the function of Lyapunov $V(\boldsymbol{x})$ one way is to assume the quadratic form: $V(\boldsymbol{x})=\boldsymbol{x}^{T} P \boldsymbol{x}$, where $P$ must be a Hermitian matrix, positively defined, that is, the self-values of the matrix $P$ must all be positive. Now, once the function $V(\boldsymbol{x})$ has been defined, the matrix P must be terminated by the following procedure:

$$
\begin{aligned}
& \dot{V}(\boldsymbol{x})=\frac{d}{d t}\left(\boldsymbol{x}^{T} P \boldsymbol{x}\right) \\
& =\boldsymbol{x}^{T} P \dot{\boldsymbol{x}}+\dot{\boldsymbol{x}}^{T} P \boldsymbol{x} \\
& =\boldsymbol{x}^{T} P(\boldsymbol{A} \cdot \boldsymbol{x})+(\boldsymbol{A} \cdot \boldsymbol{x})^{T} P \boldsymbol{x} \\
& =\boldsymbol{x}^{T}\left(P \cdot A+A^{T} . P\right) \boldsymbol{x} \\
& =-\boldsymbol{x}^{T} Q \boldsymbol{x}
\end{aligned}
$$

for which $P . A+A^{T} . P=-Q$ to guarantee asymptotic stability, it is enough that $Q$ is arbitrarily defined positive, it is convenient to choose $Q=I$, where $I$ is the identity shade. Finally, to find the elements of the matrix $P$, the elements of $P . A+A^{T} . P$ with $-Q$ are equal element by element.

\subsection{Characterization of generator stability by Lyapunov}

The power system considered in this paper, consists of a synchronous generator (Revel, León, \& Moiola, 2010) connected to infinite bus as shown in Figure 1.

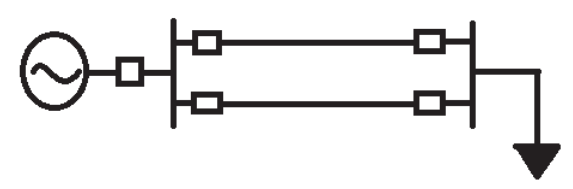

Figure 1. Generator connected to an infinite bus

The mathematical model that describes the previous system is represented by the equations:

$$
M \ddot{\delta}=P-D \dot{\delta}-\eta_{1} E_{q} \sin \delta
$$




$$
\tau \dot{E}_{q}=-\eta_{2} E_{q}+\eta_{3} \cos \delta+E
$$

where $\delta$ is the angle of the rotor in radians, $E_{q}$ is the generator voltage in p.u., $D$ is the damping coefficient, $E$ is the input voltage, $P$ is the mechanical power, $M$ is the inertial coefficient of the rotor, $\tau$ is a time constant and $\eta_{1}, \eta_{2}, \eta_{3}$ are constant parameters related to internal characteristics of the generator. (Paghi, \& Mishra, 2015)

The above equations can be rewritten to form the system state space, in the following way: Taking $\omega=\dot{\delta}$ and $\dot{\omega}=\ddot{\delta}$, therefore the system will be written using the equations of state:

$$
\begin{gathered}
\dot{\delta}=\omega \\
\dot{\omega}=\left(P-D \omega-\eta_{1} E_{q} \sin \delta\right) / M \\
\dot{E}_{q}=\left(-\eta_{2} E_{q}+\eta_{3} \cos \delta+E\right) / \tau
\end{gathered}
$$

Clearly, we can observe the non-linearity of this system of state equations, which should be easily analyzed and analyzed using computational tools such as the ode45 function available in the Matlab software. Then, each one of the curves of the state variables can be observed, once the system is solved with the following values of the parameters: $P=0.815, E=1.22, \eta_{1}=1.22, \eta_{2}=2, \eta_{3}=1.7, \tau=6.6, M=0.0147, D=0.0588$.
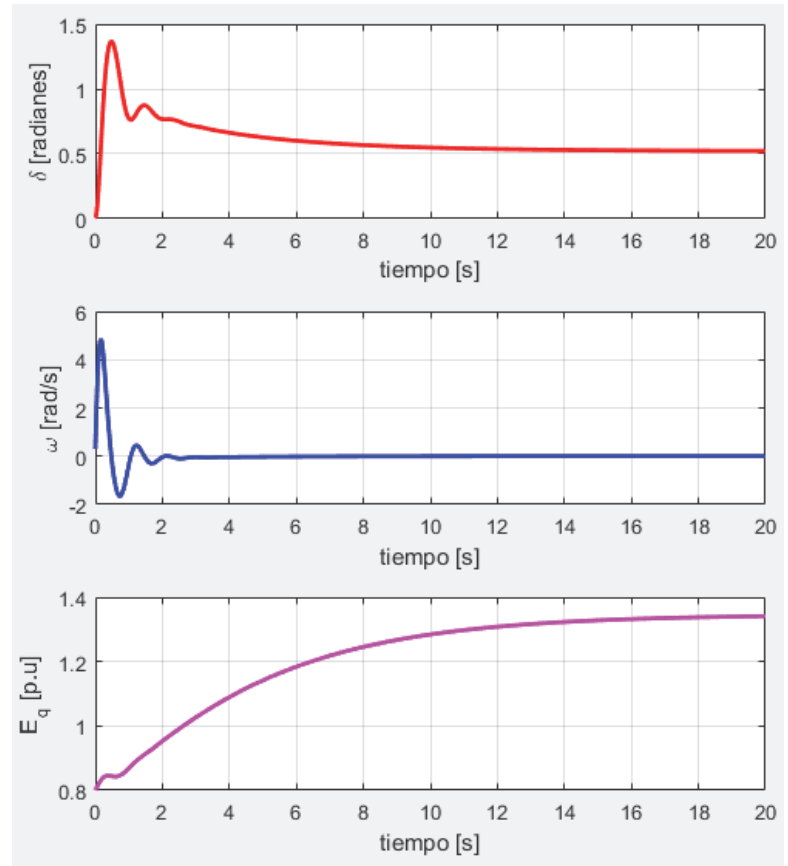

Figure 2. Synchronous generator state variables

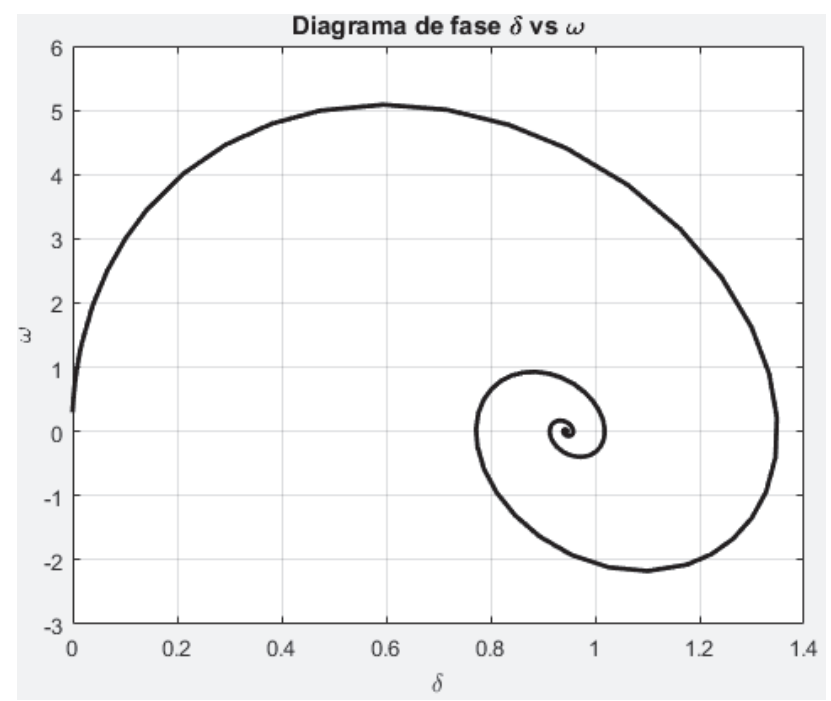

Figure 3. Phase diagram for $\delta$ vs $\omega$ 
The stability of the system occurs only if $\omega=\dot{\delta}=0$, a condition that makes the machine not lose synchronism with the network. It can be shown that this stability will depend both on the values of the parameters of the machine, such as that of the initial conditions, which can be seen in Figure 4 where the region of attraction of the state variables $\delta$ and $\omega$ is shown.

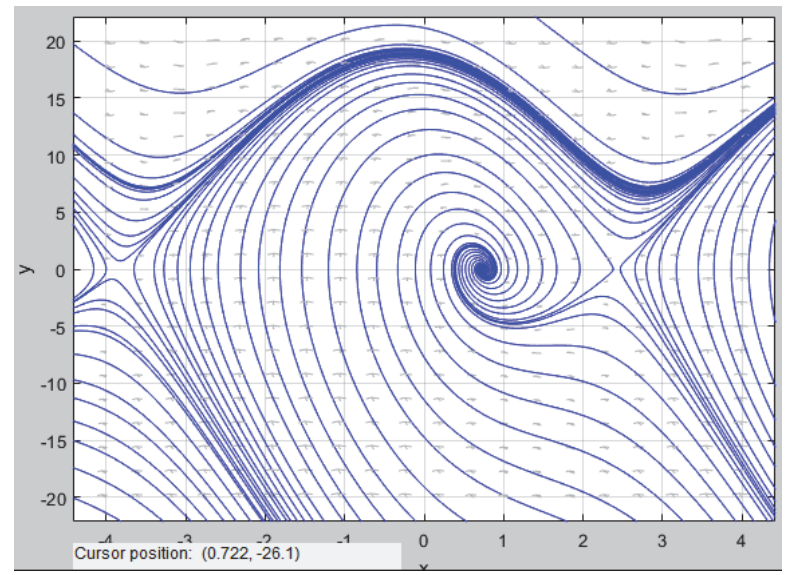

Figure 4. Region of attraction of the system

If the value of the damping coefficient $D$ is modified, the system loses stability, since $\omega$ stops being equal to zero, this case is shown in the following Fugure.
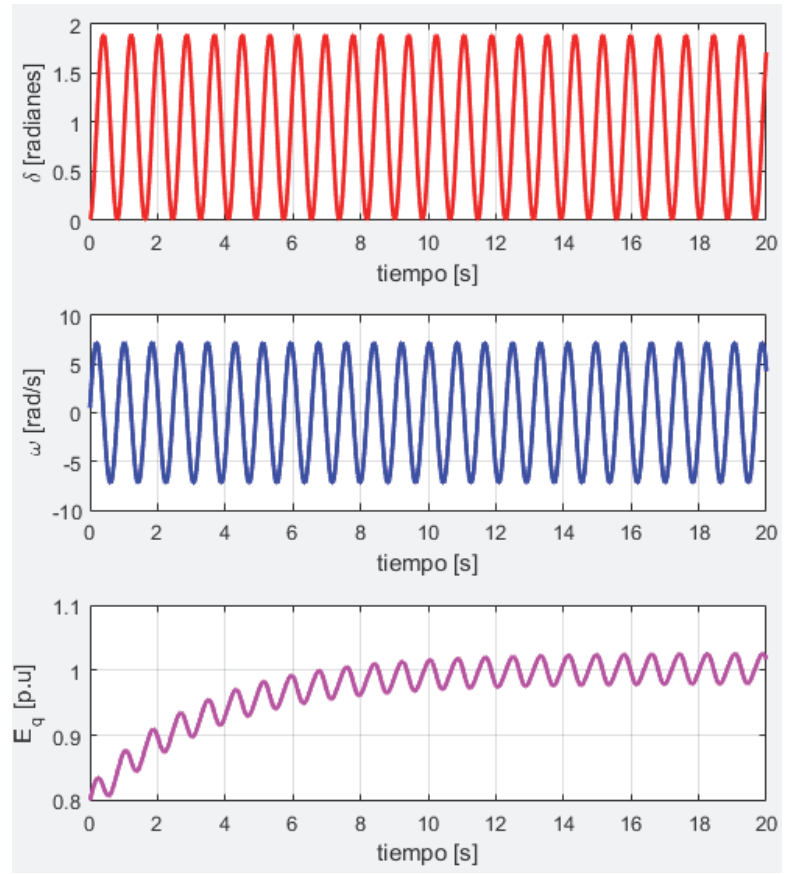

Figure 5. State variables for $D=0$

\subsection{Construction of the Lyapunov function}

For the system of state equations proposed in (3) it can be considered that the voltage of the machine remains constant and equal to 1 p.u., then the equations of state can be reduced to a system of two equations, also taking as state variables $x_{1}=\delta$ and $x_{2}=\omega$, then:

$$
\begin{gathered}
\dot{x}_{1}=x_{2} \\
\dot{x}_{2}=\frac{1}{M}\left(-\eta_{1} \sin x_{1}-D x_{2}+P\right)
\end{gathered}
$$

from the previous model and with the values of the parameters set before, we get 


$$
\begin{gathered}
\dot{x}_{1}=x_{2} \\
\dot{x}_{2}=55.44-8.99 x_{2}-4 \sin x_{1}
\end{gathered}
$$

For the construction of Lyapunov energy function, we linearize the model around its operating point:

$$
\begin{gathered}
\dot{x}_{1}=\Delta x_{2} \\
\dot{x}_{2}=\frac{1}{M}\left(-\eta_{1} \cos x_{10} * \Delta x_{1}-D * \Delta x_{2}+P\right)
\end{gathered}
$$

where $x_{10}$ and $x_{20}$ are the values of the operating point of the state variables, which are $0.3840 \mathrm{rad}$ and $0 \mathrm{rad} / \mathrm{s}$ respectively. With this linearized model we obtain the function of Lyapunov:

$$
V(x)=x^{T} P x
$$

Now, to determine the matrix $P$, we do

$$
A=\left[\begin{array}{cc}
0 & 1 \\
\frac{-\eta_{1} \cos x_{10}}{M} & -\frac{D}{M}
\end{array}\right]
$$

and then

$$
\begin{gathered}
P . A+A^{T} . P=-Q \\
{\left[\begin{array}{ll}
P_{11} & P_{12} \\
P_{21} & P_{22}
\end{array}\right] \cdot\left[\begin{array}{cc}
0 & 1 \\
-76.95 & -4
\end{array}\right]+\left[\begin{array}{cc}
0 & 1 \\
-76.95 & -4
\end{array}\right]^{T}\left[\begin{array}{ll}
P_{11} & P_{12} \\
P_{21} & P_{22}
\end{array}\right]=-\left[\begin{array}{ll}
1 & 0 \\
0 & 1
\end{array}\right]} \\
{\left[\begin{array}{cc}
-153.9 P_{12} & P_{11}-4 P_{12}-76.95 P_{22} \\
P_{11}-4 P_{12}-76.95 P_{22} & 2 P_{12}-8 P_{22}
\end{array}\right]=-\left[\begin{array}{ll}
1 & 0 \\
0 & 1
\end{array}\right]}
\end{gathered}
$$

where we obtain the system

$$
\begin{gathered}
-153.9 P_{12}=-1 \\
P_{11}-4 P_{12}-76.95 P_{22}=0 \\
2 P_{12}-8 P_{22}=-1
\end{gathered}
$$

Finally, the matrix $\mathrm{P}$ is given by

$$
P=\left[\begin{array}{ll}
9.769741 & 0.006497 \\
0.006497 & 0.126624
\end{array}\right]
$$

which is a definite positive hermitic matrix, therefore, the final Lyapunov function will be:

$$
V(\boldsymbol{x})=9.7697401 x_{1}^{2}+0.012994 x_{1} x_{2}+0.126624 x_{2}^{2}
$$

which meets the condition of stability, which can be proven by calculating the self-values of the matrix $P$, which must be positive. The solution of the linearized system can be seen in the following figure.

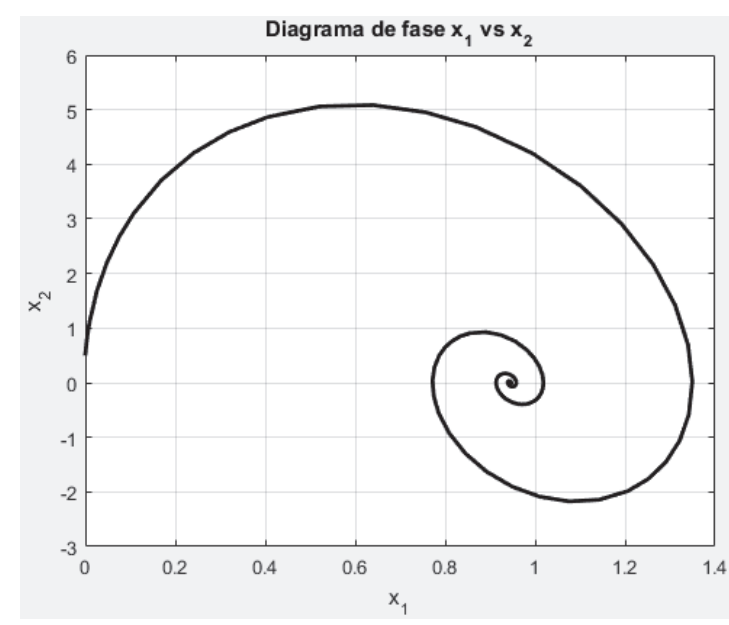

Figure 6. Phase diagram for the linearized model 


\section{Concluions}

When the value of the damping coefficient parameter is modified, a change in the dynamics of the system is obtained, which represents that there is a bifurcation for this parameter, this is a topic which can be analyzed in a broad way in other studies. There are stable systems, whose region of attraction is given by a limit cycle, these systems are oscillating systems, and their dynamics are undesirable for electrical power systems, since these oscillations cause damage to the devices, therefore, for these systems Asymptotic or fixed-point stability must be guaranteed. When a scalar function can not be established that meets the conditions necessary to be a function of Lyapunov, it is not possible to conclude on the stability of the dynamics of the system. Therefore, other algorithms must be applied for the determination of FL as is the use of partial equations.

\section{Acknowledgments}

We would like to express our sincere gratitude to Master's Programme in mathematics for the support of this research, to thank the referee for his valuable suggestions that improved the presentation of this paper and the Department of Mathematics of the Universidad Tecnolgica de Pereira (Colombia) and the group GEDNOL.

\section{References}

Khalil, H. (1996). Nonlinear systems. Prentice-Hall (2nd ed).

López, F., Sánchez, T., \& Moreno, J. (2014). Construcción de Funciones de Lyapunov para sistemas Homogéneos de segundo Orden. Memorias del XVI congreso Latinoamericano de control automático, CLCA. http://132.248.52.100:8080/xmlui/handle/132.248.52.100/7345

Malisoff, M., \& Mazenc, F. (2009). Constructions of Strict Lyapunov Functions. Communications and Control Engineering, 1 .

Paghi, S., \& Mishra. B. (2015). Solution of swing equation for transient stability analysis in dual-machine system (pp. 34-39). IOSR Journal of Engineering, 5.

Revel, G., León, A., Moiola, J., \& Anderson, C. A. (2010). Bifurcation analysis on a detailed multimachine power system model (pp. 937-949). IEEE Transactions on Circuits and Systems I: Regular Papers. Vol. 57. http://10.1109/TCSI.2009.2026686

Seron, M. (2000). Sistemas no lineales. Universidad Nacional del Rosario. Colombia.

Strogatz, S., \& Fox, F. (1995). Nonlinear dynamics and chaos: With applications to physics, biology, chemistry and engineering (pp. 93). Physics Today, 48.

\section{Copyrights}

Copyright for this article is retained by the author(s), with first publication rights granted to the journal.

This is an open-access article distributed under the terms and conditions of the Creative Commons Attribution license (http://creativecommons.org/licenses/by/3.0/). 